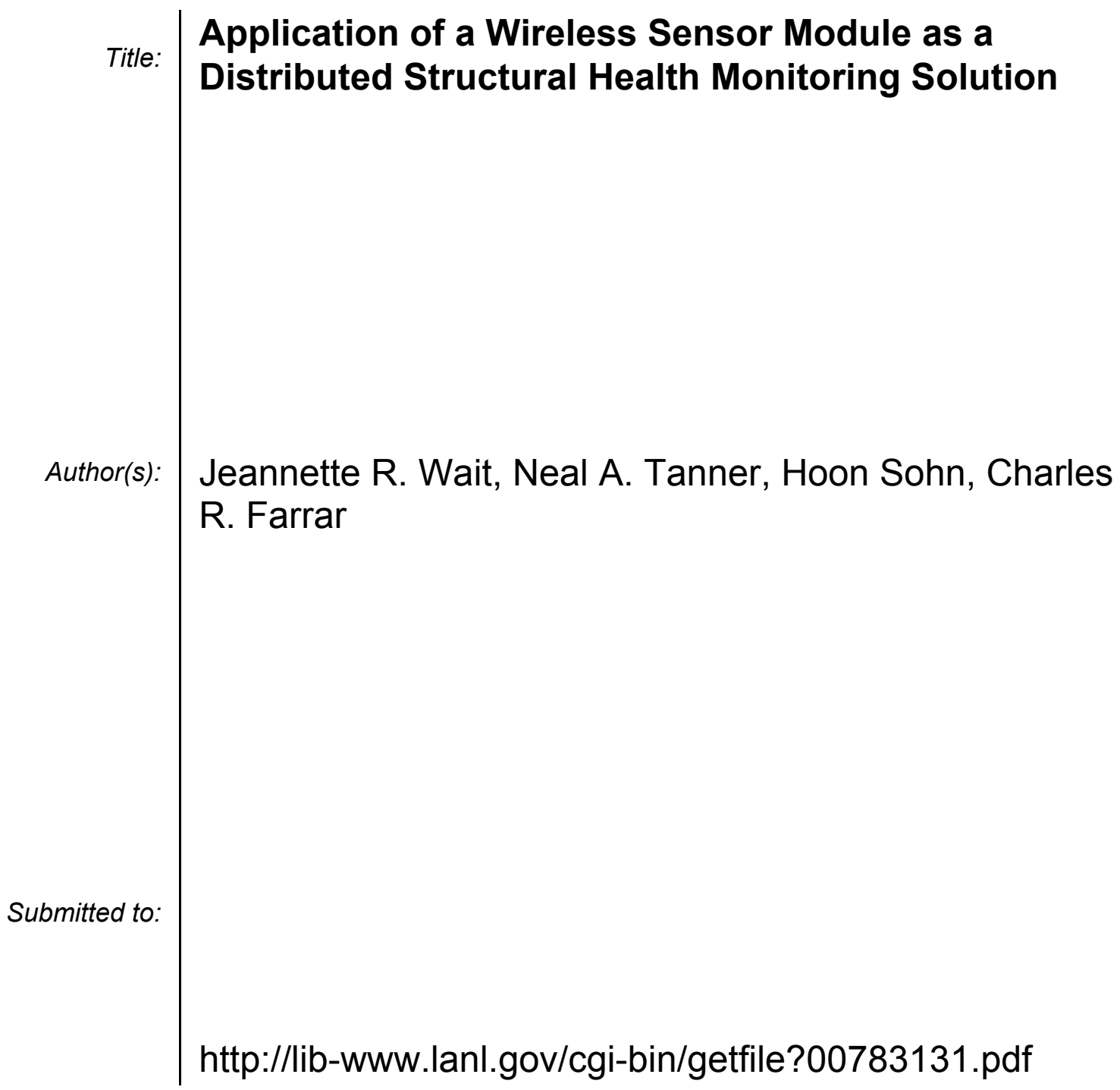




\title{
Application of a Wireless Sensor Module as a Distributed Structural Health Monitoring Solution
}

\author{
Jeannette R. Wait ${ }^{1}$, Neal A. Tanner ${ }^{2}$, Hoon Sohn ${ }^{3}$, Charles R. Farrar ${ }^{4}$ \\ ${ }^{I}$ Department of Civil Engineering, University of Southern California, Los Angeles, CA 90089, USA \\ ${ }^{2}$ Department of Mechanical Engineering, Stanford University, Palo Alto, CA 94305, USA \\ ${ }^{3,4}$ ESA-WR, Los Alamos National Laboratory, Los Alamos, NM 87545, USA
}

\begin{abstract}
A damage detection system was developed with commercially available wireless sensors. To showcase the capabilities of the damage detection system, a portable demonstration structure was constructed to monitor joint failure induced on one joint of the structure by a piezoelectric actuator. Two damage detection algorithms were used to identify joint damage. First, a statistical process control method was used to monitor the correlation of two accelerometer readings mounted across a joint. Then, a combination of time series analysis and sequential hypothesis test was used to classify a joint as damaged or undamaged.
\end{abstract}

\section{INTRODUCTION}

The goal of this study was to develop a robust and cost-effective local structural health monitoring solution, which integrates wireless transmitter, micro-electromechanical systems (MEMS) sensors, onboard processors, and damage detection algorithms. For this project, emphasis was placed on adapting the structural health monitoring system to the limitations of off-the-shelf wireless sensing and data processing hardware because of the focus towards a proof-of-concept rather than designing a field installable product. A wireless hardware system called the "motes" manufactured at Crossbow Inc. (www.xbow.com) and running UC Berkeley's TinyOS operating system (tinyos.millennium.berkeley.edu) was chosen because of their ready-made wireless communication capabilities. The effectiveness of this integrated system unit is demonstrated using a portal structure with bolted joints, where damage is introduced by releasing preload in a joint.

\section{WIRELESS SYSTEM}


TinyOS is built on top of the $\mathrm{C}$ programming language and provides the programmer with pre-packaged functionality for interfacing with hardware components such as the ADC. All sensor programming for this project was done within the confines of TinyOS.

Two ADXL202 accelerometers, which are commercially available from Analog Devices Inc., are used for this study. The ADXL202 is a dual axis, $\pm 2 \mathrm{~g}$ MEMS accelerometer and the sensor board's original configuration was modified by changing capacitors to increase the bandwidth of the accelerometer from the original $50 \mathrm{~Hz}$ to $1 \mathrm{kHz}$.

The core of the processor board is a $4 \mathrm{MHz}$ ATMEL AVR 90LS8535 microprocessor with 8 $\mathrm{KB}$ of flash program memory and 512 bytes of RAM (see Figure 1). A 10-bit analog-todigital converter (ADC) is included in the microprocessor. The processor board also contains three LED lights and a short range $916 \mathrm{MHz}$ radio transmitter.

Programs were written on a PC and compiled into a binary image file that was downloaded into the flash program memory on the processing board. This firmware download was accomplished by placing the processing board in a programming bay connected through the PC's parallel port (see Figure 2). The serial port is also used for data transfer from the processing board back to the PC.

Two independent accelerometers were connected to a single processing board. A simple crossover in one of the cables allowed the two accelerometers to be accessed through separate channels on the ADC. In this manner, a single processing board could collect data from up to 4 channels, perform basic digital signal processing on the measured time histories, and broadcast the processed data over the radio link. A second processing board situated in the programming bay received broadcast data and relayed it to the PC.

The ADC itself can handle sampling rates of up to $4 \mathrm{kHz}$, but the achievable sampling rates were dictated by either the radio bandwidth (when transmitting raw data) or the processing power of the microprocessor and typically ranged between $256 \mathrm{~Hz}$ and $1 \mathrm{kHz}$. The system had a full-scale range of $\pm 2 \mathrm{~g}$ with a resolution of $17 \mathrm{mg}$. Both accelerometers and ADCs showed considerable DC bias and required calibration for each combination of a particular accelerometer and ADC.

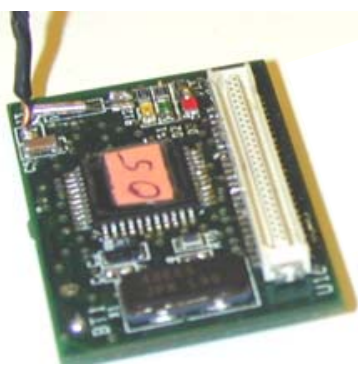

Figure 1: Processing board with wireless transmitter

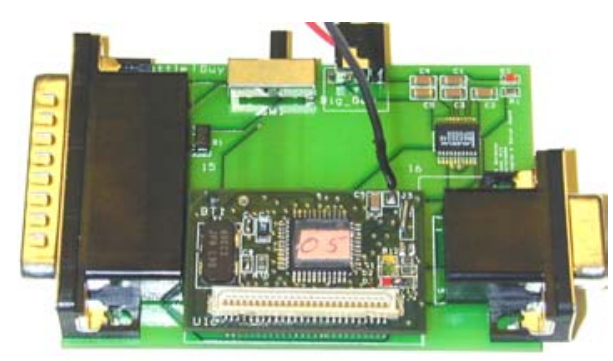

Figure 2: Programming bay 


\section{DAMAGE DETECTION ALGORITHMS}

\subsection{Statistical Process Control}

The first feature used was the cross correlation coefficient $\tau_{n}$ between time history data from two accelerometers mounted across a joint:

$$
\tau_{n}=\sum_{i=1}^{n} x_{i} \times y_{i} / n
$$

where $x_{i}$ and $y_{i}$ are the time series from two accelerometers. The packet size $n$ was typically 32 . This approach could only be implemented at a sampling rate of $256 \mathrm{~Hz}$. The lack of sufficient memory to log an entire packet's worth of data forced the use of "on-thefly" algorithms that significantly decreased the attainable sampling rates.

Once $\tau_{n}$ had been calculated for a packet of data, the sample mean $\mu$ and the variance $\sigma^{2}$ of $\tau_{n}$ were continuously updated, and used to establish control limits of a statistical process control. Upper and lower limits were set at $\mu \pm 2.5 \sigma$.

Because of time, memory, and processing constraints, the computation of $\mu$ and $\sigma^{2}$ was performed on the laptop. After the bounds were calculated on the laptop, they were hardcoded back onto the processing board.

After the training was conducted using the baseline time series, the cross correlation features were calculated for new time histories in exactly the same manner as in the training phase, but were then monitored against the bounds established in the training phase.

When an outlier was detected, the occurrence of the outlier was registered in a binary variable $T . T$ takes a value of unity when there is an outlier. Otherwise, $T$ is set to zero. In addition, the damage measure $S_{n}$ was defined as:

$$
S_{n}=S_{n-1} \times \frac{24}{25}+T \times 40
$$

The numerical values in Equation (2) were tailored so that $S_{n}$ ranges from 0 to 1000 . In the current monitoring program, the red LED signals joint damage when $S_{n}$ exceeds 100 . For instance, an evenly spaced outlier out of every 12 th points or 3 or more successive outliers will signal the red LED. A warning message can also be transmitted back to the base station.

\subsection{Time Series Analysis-Sequential Hypothesis Test}

A combination of time series analysis and sequential hypothesis test is presented as a more sophisticated damage classifier. First, a time prediction model called AR-ARX model [Sohn and Farrar 2001] was fit to the point-by-point difference between two accelerometer readings near a single joint. The approach was based on the premise that, when the time series model was constructed from the baseline time series and was used to predict data from a damaged case, the time series model could not capture the underlying dynamics of the damaged system and the prediction error would increase. Based on this assumption, a hypothesis test called a sequential probability ratio test (SPRT) [Allen et al. 2002] was conducted on the prediction errors to determine the condition of the joint: 


$$
H_{o}: \sigma\left(\varepsilon_{y}\right) \leq \sigma_{o}, \quad H_{1}: \sigma\left(\varepsilon_{y}\right) \geq \sigma_{1}, \quad 0<\sigma_{o}<\sigma_{1}<\infty
$$

When the standard deviation of the residual error $\sigma\left(\varepsilon_{y}\right)$ is less than a user specified lower bound $\sigma_{o}$, the null hypothesis is accepted and the system in question is considered undamaged. On the other hand, when $\sigma\left(\varepsilon_{y}\right)$ becomes equal to or larger than the other user specified upper bound $\sigma_{1}$, the null hypothesis is accepted and the system is suspected to be damaged. For this hypothesis test, a SPRT, $\mathrm{S}(b, a)$, makes three distinctive decisions at stage $n$ (Ghosh, 1970);

$$
\begin{aligned}
& \text { (1) Accept } H_{o} \text { if } Z_{n} \leq \ln \frac{\beta}{1-\alpha} \\
& \text { (2) Reject } H_{o} \text { if } Z_{n} \geq \ln \frac{1-\beta}{\alpha}, \\
& \text { (3) Continue observing data if } \ln \frac{\beta}{1-\alpha} \leq Z_{n} \leq \ln \frac{1-\beta}{\alpha}
\end{aligned}
$$

where the transformed random variable $Z_{n}$ is the natural logarithm of the probability ratio at stage $n . \alpha$ and $\beta$ the pre-determined upper limits for type I and II errors, respectively.

\section{EXPERIMENTAL STUDY}

\subsection{Description of Experiments}

A bolted frame structure shown in Figure 3 was used as a test bed for this study. Two different configurations of the experiment were used for the demonstration of the mote system and for the conventional data acquisition system. Note that, because the computation power of the mote system was limited to fully demonstrate the capability of the damage detection algorithms developed at LANL, an additional experiment was conducted using the conventional wired data acquisition system to highlight the potential of the damage detection algorithms. Hereafter, the experimental configuration related to the mote demonstration is referred to Experiment 1 (see Figure 3), and the one associated with the conventional hardware system is called Experiment 2 (see Figure 4).

A piezoelectric (PZT) actuator was used to change the bolt preload of a joint without disturbing the structure. By varying the input voltage to the actuator from $-200 \mathrm{~V}$ to +1000 $\mathrm{V}$, a $4 \mathrm{kN}$ change in the bolt tension could be achieved. The PZT actuator allows for modeling gradual degradation or deterioration of a structural system. For the first experiment, the voltage was manually controlled and a pulse generator was used for the second experiment. For the demonstration of the mote system, two Analog Device MEMS accelerometers with $312 \mathrm{mv} / \mathrm{g}$ sensitivity were used, and placed across the actuated joint. For the conventional wired test, four PCB ICP accelerometers with $10 \mathrm{mv} / \mathrm{g}$ were used.

An electromagnetic shaker was used to excite the structure. For Experiment 1 shown in Figure 3, the structure was excited horizontally near the base with a $100 \mathrm{~Hz}$ sin wave, and the input to the shaker was generated using a virtual signal generator in MATLAB ${ }^{\circledR}$. For Experiment 2 in Figure 4, the shaker was suspended by bungee cords from a small frame built next to the test structure to remove undesired feedback forces from the test structure. Also, a signal generator provided a random input to the shaker after being amplified. The 
random signal had a bandwidth approximately equal to $4 \mathrm{kHz}$ and was 0.28 volts in amplitude.

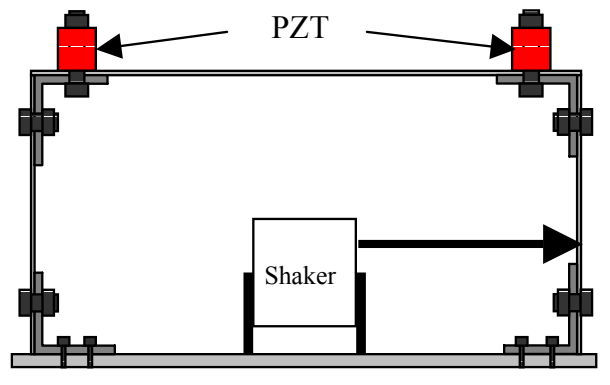

Figure 3: Experiment 1 setup

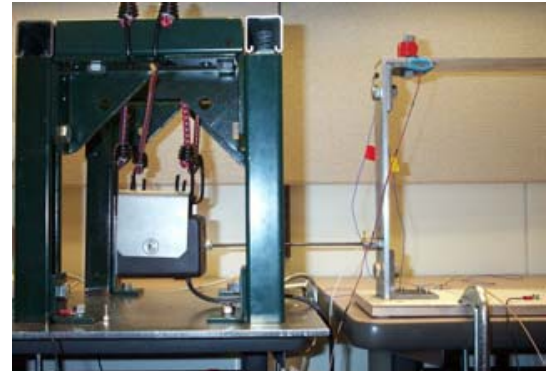

Figure 4: Experiment 2 setup

\subsection{Diagnosis Results}

\subsubsection{Application of Statistical Process Control}

With the bolt in the fully tight condition, baseline data were collected to establish the control limits. In this configuration, a sample mean of -3.5 and a standard deviation of 5.0 were obtained resulting in upper and lower bounds of -18.5 and +11.5 , respectively.

The monitoring phase started with the actuator at $+1000 \mathrm{~V}$. The voltage was then quickly reduced to $-200 \mathrm{~V}$, and was brought back to the initial voltage. As shown in Figure 5, when the voltage was reduced to the minimum value, the monitoring program successfully identified the decrease of the correlation between the two sensors in a joint and signaled the red LED accordingly.

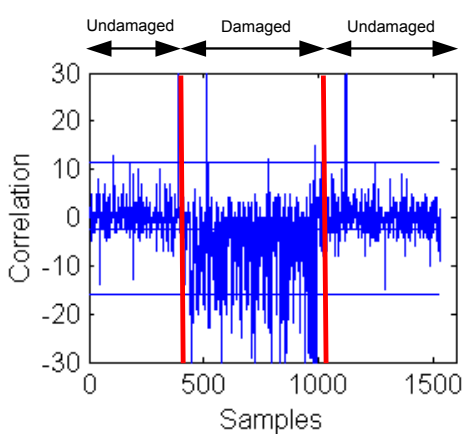

Figure 5:Change in correlation values caused by reducing and then increasing the preload in the bolted connection on the demonstration structure

\subsubsection{Application of Combined AR-ARX \& SPRT Algorithm}

The combined AR-ARX/SPRT algorithm was demonstrated using data measured from the conventional wired data acquisition system. Twenty-six runs were taken for both the training 
and test phases. During the training phase, the actuator voltage was set to $+1000 \mathrm{~V}$ to simulate the tight condition of the bolt, and the gradual loss of preload in one of the joints was simulated during the testing phase.

Following the procedure in Section 3.2, the SPRT was performed. An example of the SPRT plot corresponding to the gradual damage in Joint 1 is shown in Figure 6. For the case shown, $\sigma_{o}$ and $\sigma_{1}$ in Equation (3) were set to $2.8 \sigma\left(\varepsilon_{x}\right)$ and $3.0 \sigma\left(\varepsilon_{x}\right)$, respectively. $\sigma\left(\varepsilon_{x}\right)$ is the standard deviation of the baseline prediction errors. This figure demonstrates that, as time goes on, the Z-statistic for Joint 1 moves farther and farther away from the upper limit indicating that the joint is damaged. When the SPRT is applied to the undamaged case, the Z-statistic crosses the lower limit very quickly inferring that the joint is undamaged.

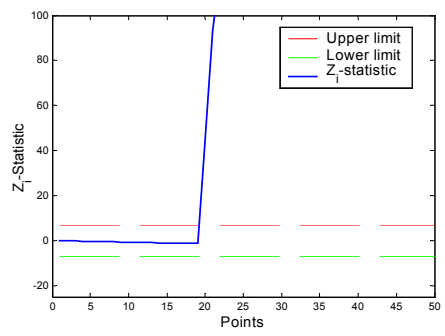

Figure 6: An example of the SPRT where the $\mathrm{Z}$ statistic crosses the upper limit indicating the decrease of the bolt preload

\section{SUMMARY}

In this study, a proof-of-concept wireless monitoring system was developed from commercially available wireless sensing components. The monitoring system was applied to a bolted frame structure specifically designed such that preload in the bolted connections could be modified without introducing extraneous inputs such as those caused by the impact of a wrench applied to the joint.

Within the hardware limitations of this off-the-shelf system the monitoring system was shown to successfully identify changes in the preload of a bolted connection. This damage was detected by implementing simplified statistical pattern recognition algorithms onto the local processor. In addition, due to hardware limitations of the wireless module, a conventional data acquisition system was used to implement a second, more robust, damage identification algorithm. Both damage identification algorithms were successful in detecting and locating a damaged bolted joint.

Due to the approach of using off-the-shelf hardware, several very serious issues arose that greatly complicated or impeded the development of the remote structural health monitoring system. The first of these limitations arose out of the very limited range and resolution of the sensing system itself. The range was limited by the accelerometer itself while the resolution was limited by only having a 10 bit ADC. Because of the small size of the flash program memory, any programs that contained floating-point calculations would not fit into the flash. Having to perform all processing in integer format led to some very harsh tradeoffs between range and precision of calculations. In addition, because a large programmable memory 
space was required on the microprocessor for implementing the AR-ARX and SPRT algorithms, a traditional data acquisition system was used to demonstrate the ARARX/SPRT algorithm.

\section{REFERENCES}

Allen, D. W., et al., (2002) "Utilizing the Sequential Probability Ratio Test for Building Joint Monitoring, " Proceedings of SPIE NDE Conference, San Diego, CA, March 17-21, 2002.

Sohn, H., and Farrar, C. R., (2001) "Damage Diagnosis Using Time Series Analysis of Vibration Signals, " Journal of Smart Materials and Structures 10, pp. 446-451.

University of California, Berkeley TinyOS Project, (2002) "TinyOS: An Operating System for Networked Sensors," <http://tinyos.millennium.berkeley.edu>. 